\title{
Artigo Original / Original Paper \\ Etnobotânica como subsídio à gestão socioambiental de uma unidade de conservação de uso sustentável
}

\author{
Ethnobotany as a tool for the socio-environmental management \\ of a sustainable use protected area
}

\author{
Amanda C.C. Prado ${ }^{1}$, Eliane B. Rangel ${ }^{1}$, Hildeberto C. de Sousa ${ }^{1}$ \& Maria Cristina T.B. Messias ${ }^{1,2}$
}

\begin{abstract}
Resumo
A etnobotânica é útil para compreender a relação dos povos e plantas, facilitando a proposição e implementação de estratégias de melhoria da qualidade de vida e de conservação ambiental. Portanto, investigou-se o conhecimento etnobotânico na área de proteção ambiental (APA) Cachoeira das Andorinhas em Ouro Preto-MG. Foram realizadas entrevistas semiestruturadas e turnês-guiadas para o levantamento das plantas utilizadas e do contexto socioambiental dos usuários. As espécies foram categorizadas pelo uso. A riqueza de espécies conhecidas entre os diferentes grupos de usuários e entre categorias utilitárias foi comparada pelo teste G. Registrou-se 232 espécies para 10 tipos de uso, sendo a maioria medicinais e alimentares. Mulheres conhecem mais plantas medicinais e homens, madeireiras. Cerca de 50\% das espécies utilizadas são exóticas, sobressaindo-se as frutas utilizadas no preparo de doces, atividade tradicional do distrito. As áreas antropizadas são as mais exploradas para obtenção dos recursos, seguida pelas florestas. A tradição secular, o rico conhecimento e a dependência da comunidade no uso das plantas sugerem a sustentabilidade do uso. Propõe-se ações para garantir a consolidação dos objetivos dessa unidade de conservação. Palavras-chave: áreas protegidas, comunidades tradicionais, conhecimento tradicional, conservação da biodiversidade, gestão de unidades de conservação.
\end{abstract}

\begin{abstract}
Ethnobotany is useful for understanding the relationship between people and plants, facilitating the suggestion and implementation of strategies to improve the quality of life and environmental conservation. Thus, an ethnobotanical study was carried out in the environmental protection area Cachoeira das Andorinhas in Ouro Preto, Minas Gerais, Brazil. Semi-structured interviews and guided tours were performed to survey the plants used and the socioeconomic user profiles. The species were categorized according to their use. The richness of known species among the user groups and utilitarian categories was compared using the G-test. There were 232 species for 10 types of use, most of them are medicinal and edible. Women know more medicinal plants and men, timber species. The ancient tradition, the rich knowledge and the dependence of the community on the plants suggest the sustainability of the use. Actions are proposed to ensure the consolidation of the protected area objectives.

Key words: conservation areas, traditional communities, traditional knowledge, biological conservation, park management.
\end{abstract}

\section{Introdução}

Unidades de conservação (UC) de uso sustentável preveem a possibilidade de utilização de recursos, desde que não comprometam os objetivos e a integridade ambiental (MMA 2004).
No entanto, no Brasil, nem sempre estes critérios de uso sustentável são claros, e muitas dessas UC's carecem de planos de manejo. Desta forma, na gestão dessas UC's frequentemente ocorrem restrições de uso dos recursos, semelhantes àquelas

\footnotetext{
${ }^{1}$ Universidade Federal de Ouro Preto, Depto. Biodiversidade, Evolução e Meio Ambiente, Campus Universitário do Morro do Cruzeiro s/n, Ouro Preto, MG, Brasil.

${ }^{2}$ Autor para correspondência: cristinabotanica@gmail.com
} 
das unidades de proteção integral (Maia-Neto 2009). Se por um lado a restrição do uso dos recursos naturais tem importância na preservação da biodiversidade, por vezes, ela impõe alterações no modo de vida da comunidade, pela limitação de atividades antes praticadas. Este fato gera conflitos com as comunidades que habitam essas áreas protegidas.

O uso sustentável de recursos, além de preservar a cultura e trazer benefícios socioeconômicos às comunidades, é também um aliado à conservação ambiental. Ostrom (1990) descreve os recursos naturais como bens públicos e de acesso comum, com possibilidade de autonomia da comunidade para a gestão do uso sustentável.

Muitos povos associam o uso dos recursos naturais à sua conservação, pois têm uma dependência direta desses recursos para a sua subsistência, desenvolvimento econômico e social (Diegues \& Viana 2004; Hegde \& Enters 2000; Lima et al. 2012). Vários autores têm proposto formas de se avaliar a interação destas populações com os recursos naturais disponíveis (Berkes 1999; Hanazaki 2003; Souza \& Kubo 2006; Albuquerque et al. 2010). Neste âmbito, estudos etnobotânicos representam uma importante ferramenta de diagnóstico para a gestão socioambiental, gerando informações úteis ao planejamento estratégico de ações políticas e educativas voltadas para a conservação e manejo sustentável de recursos naturais (Diegues \& Viana 2004). Dentre essas informações relevantes geradas pelos estudos etnobotânicos podem ser citadas aquelas relativas à diversidade e composição das espécies utilizadas e de suas interações com os atributos socioeconômicos e culturais da população usuária (Albuquerque 2006; Sousa et al. 2012; Campos et al. 2015). Deste modo, pode-se inferir se diferenças de gênero, idade, renda, nível de escolaridade, ou outra característica da comunidade interfere no saber tradicional com relação ao uso dos recursos. A idade é uma variável comumente associada ao saber tradicional e diversos estudos demonstram a preponderância dos mais velhos como maiores detentores do conhecimento sobre plantas (AriasToledo et al. 2007; González et al. 2011; Campos et al. 2015; Messias et al. 2015). A questão do gênero sobre a distribuição do conhecimento é também muito discutida em etnobotânica. Muitos estudos tem demonstrado que homens e mulheres concentram o conhecimento em categorias de uso de plantas distintas devido à diferenças nos papéis sociais desempenhados de acordo com o gênero
(Voeks \& Leony 2004; Arias-Toledo et al. 2007; Voeks 2007). Estudos etnobotânicos também facilitam a compreensão dos critérios de escolha de plantas para compor o elenco de espécies úteis de uma determinada localidade como, por exemplo, de espécies nativas ou exóticas, anuais ou perenes, ou ainda com diferentes categorias de uso (Albuquerque 2006; Soldati \& Albuquerque 2012; Sousa et al. 2012; Medeiros 2013). Esse arcabouço de conhecimentos a respeito das dimensões do saber e uso das plantas pelos diferentes grupos humanos é fundamental para orientar o manejo sustentável das unidades de conservação no sentido de preservação da cultura e dos recursos vegetais.

Com vistas a proposição de estratégias para consolidação dos objetivos de uma unidade de conservação de uso sustentável, o presente artigo apresenta o estudo etnobotânico da Área de Proteção Ambiental Estadual da Cachoeira das Andorinhas, analisando a relação entre seus moradores e o conhecimento tradicional sobre os recursos vegetais.

\section{Material e Métodos}

Área de estudo

A Área de Proteção Ambiental (APA) Estadual da Cachoeira das Andorinhas, com 18.700 ha (Fig. 1), abrange todo o distrito de São Bartolomeu (20'18'54"S, 4334'41"O), em Ouro Preto, Minas Gerais, cidade patrimônio mundial. Essa UC foi criada em 1989 com objetivos de proteger a nascente do Rio das Velhas, a biodiversidade local e a paisagem que emoldura o município de Ouro Preto. Dentro dos limites dessa APA, em 2003, foi criada a Floresta Estadual do Uaimií (FLOE), com objetivo de uso múltiplo e comunitário de seus recursos. A FLOE possui Plano de Manejo, porém os moradores ainda não têm acesso aos recursos do local. A APA, até o momento, não possui Plano de Manejo.

O distrito de São Bartolomeu possui uma área de $162 \mathrm{~km}^{2}$ e está localizado na transição entre a Mata Atlântica e o Cerrado, onde ocorrem florestas montanas, florestas estacionais semideciduais, florestas ripárias, campos rupestres e zonas antropizadas (IEF 2006; Scalco 2009). O relevo é acidentado, com altitudes variando entre 700 e $1.750 \mathrm{~m}$. O clima, segundo a classificação de Köppen, é Cwb, mesotérmico úmido (Álvares et al. 2013). A temperatura média anual é de cerca de $20^{\circ} \mathrm{C}$ e a precipitação anual varia de 1.300 a 1.900 $\mathrm{mm}$, com período chuvoso de outubro a março (Scalco 2009). 
A sede do distrito, onde foi realizado o estudo, possui 729 habitantes (IBGE 2010). Fundado no ano de 1724 , foi importante centro minerador para as jazidas de ouro e pedras preciosas, às margens do Rio das Velhas (IEF 2006). As atividades agrícolas no distrito datam da sua criação, principalmente o cultivo de olerícolas e frutícolas. Na década de 80 registrou-se a ocorrência de fitopatógenos de solo (Sclerotium spp.) causando doenças que limitam até hoje o cultivo de muitas olerícolas (Prado 2014). Em meados do século XX, a fabricação de doces artesanais, em especial da goiabada, passou a ter grande importância na complementação da renda familiar (IEF 2011). O processo de fabricação artesanal de doces foi registrado como o primeiro patrimônio imaterial do município de Ouro Preto (Decreto No 1.096 de 15 de Abril de 2008). Apesar da prática de fabricação de doces possuir valor cultural e conferir oportunidades de trabalho e renda, desde a criação da APA, a retirada de lenha para alimentar as fornalhas tornou-se proibida, gerando conflitos entre a comunidade e a gestão da APA (Prado 2014).

\section{Aspectos éticos e legais}

O estudo foi submetido e autorizado pelo Comitê de Ética em Pesquisa (CAAE 05301712.4.1001.5150). O termo de consentimento livre esclarecido foi utilizado para a anuência dos entrevistados para a participação nesse estudo. A licença para coleta de plantas foi obtida no Instituto Estadual de Florestas (IEF-MG).

\section{Levantamento etnobotânico}

A seleção dos informantes foi realizada na população urbana da sede do distrito de São Bartolomeu, de forma sistemática, conforme a metodologia proposta pelo IBGE (1983).

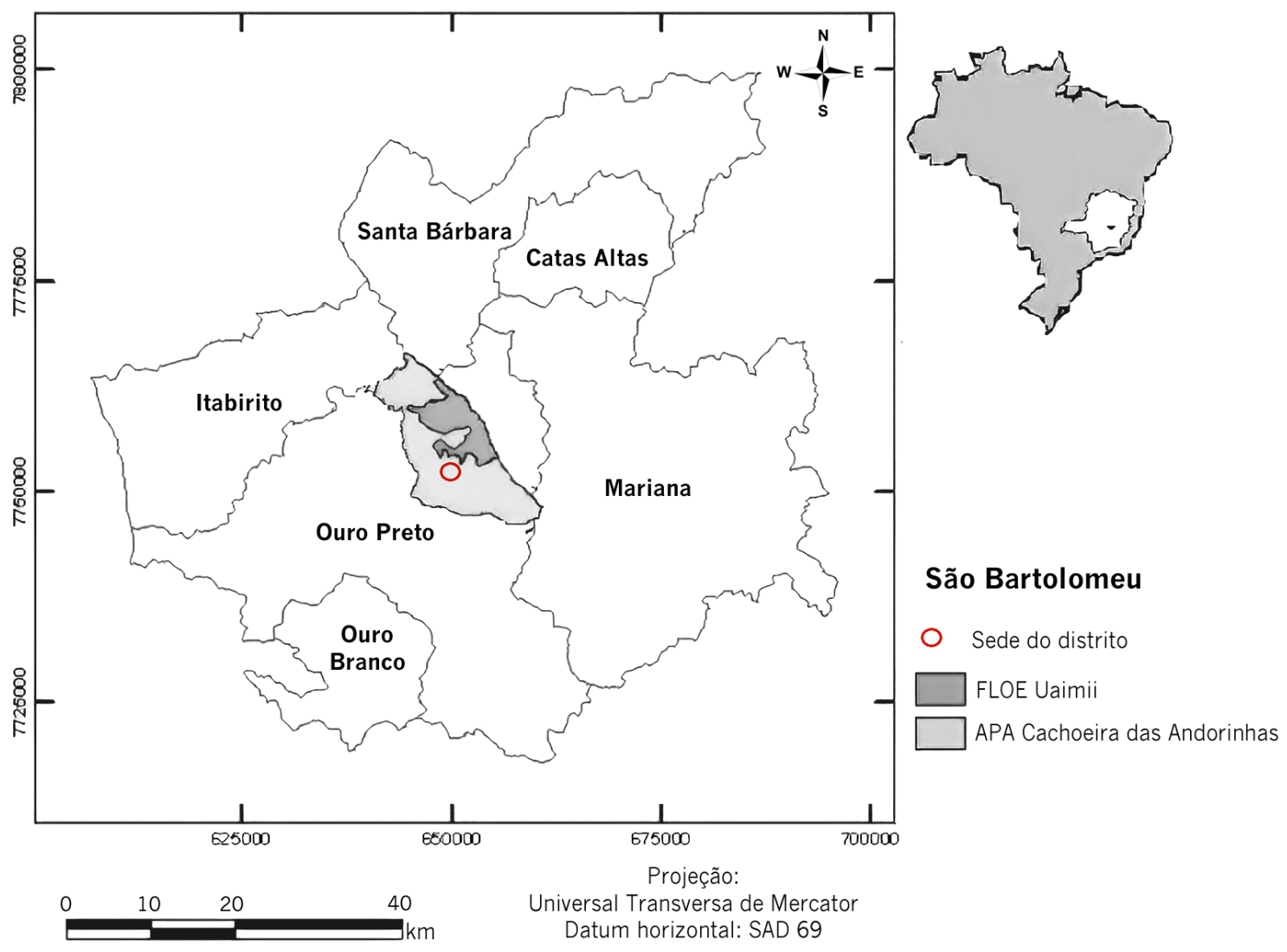

Figura 1 - Localização da área de estudo, na Área de Proteção Ambiental da Cachoeira das Andorinhas, na sede do distrito de São Bartolomeu, Ouro Preto, Minas Gerais, Brasil.

Figure 1 - Study area location, in the Cachoeira das Andorinhas Park, in the São Bartolomeu district headquarters, Ouro Preto, Minas Gerais, Brazil. 
Amostrou-se 50\% das residências de cada rua, em intervalos regulares, sorteando-se a residência inicial. Em caso de ausência dos moradores, prosseguiram-se as entrevistas na próxima moradia. Foram entrevistados todos os residentes presentes, maiores de 18 anos que concordaram com a participação. Os horários das visitações foram previamente estabelecidos, permitindo a participação de todos os residentes.

Foram realizadas entrevistas semiestruturadas contendo informações socioeconômicas (gênero, idade, tempo de moradia, escolaridade, renda e ocupação) e sobre os recursos vegetais utilizados. Para a coleta de dados sobre as espécies vegetais foram utilizadas listas livres, além de turnês guiadas e observação direta (Albuquerque et al. 2010). As espécies foram identificadas, herborizadas, depositadas no herbário Professor José Badini (OUPR) e classificadas em famílias de acordo com o APG III (Chase \& Reveal 2009). Para cada espécie citada foram registrados o nome popular, categoria de uso, parte usada e habitat. Os usos das espécies foram categorizados em medicinal, alimentar, madeireiro, ornamental, combustível, tóxico, forrageiro, artesanal, corante e místico. Verificou-se o estado de conservação das espécies em listas de espécies ameaçadas (COPAM 1997; MMA 2014; IUCN 2015).

\section{Análise dos dados}

A suficiência amostral foi verificada pelos estimadores Jackknife de primeira e segunda ordens (Magurran 1988). Foram feitas comparações quanto à riqueza de espécies vegetais conhecidas pelos usuários com diferentes categorias socioeconômicas pelo teste $\mathrm{G}$ de Williams (Zar 1996). Para tal, as características socioeconômicas foram categorizadas nas seguintes classes: a) gênero (masculino e feminino); b) idade ( $<25$ anos, 25 a 44 anos, 45 a 64 anos e $>65$ anos); c) escolaridade (analfabeto, ensino fundamental incompleto, ensino fundamental completo, ensino médio e ensino superior); d) tempo de moradia ( $\leq$ 10 anos, 11 a 30 anos, 31 a 50 anos, > 50 anos), ocupação (relacionada e não relacionada ao uso de plantas) e renda ( $\leq 1$ salário mínimo, 1,1 a 2 salários mínimos, $>2$ salários mínimos). $\mathrm{O}$ valor de uso das espécies foi calculado pela frequência de citações das mesmas, de acordo com Tardio \& Santayana (2008). A diversidade de espécies utilizadas pela comunidade foi estimada pelos índices de Shannon-Wiener ( $\left.\mathrm{H}^{\prime}\right)$ e equabilidade de Pielou (J) (Magurran 1988).

\section{Resultados e Discussão}

Levantamento etnobotânico

Foram entrevistadas 43 pessoas, sendo 27 mulheres e 16 homens. Foram registradas 232 espécies vegetais, reunidas em 74 famílias (Tab. 1, disponibilizada no link < https://doi.org/10.6084/ m9.figshare.7853402.v1>). A suficiência amostral mostrou-se satisfatória, uma vez que os estimadores Jackknife de $1^{\text {a }}$ e $2^{\text {a }}$ ordens indicaram que foram amostradas mais de $93 \%$ do total de espécies esperadas. A categoria de uso medicinal apresentou o maior número de espécies (119), 44 delas indicadas também para outros fins. A categoria alimentar teve um grande número de espécies (80) e o maior número de citações. Em escala decrescente de número de espécies, também foram identificadas plantas com uso nas categorias madeireira, ornamental, combustível, artesanal, místico, forragem, tóxico e corante (Fig. 2).

As famílias com maior riqueza de espécies foram Asteraceae (25), Fabaceae (16), Solanaceae (14), Lamiaceae (13), Myrtaceae (12) e Poaceae (11) (Tab. 1, disponibilizada no link <https://doi. org/10.6084/m9.figshare.7853402.v1>). Essas

famílias são frequentemente referidas como muito

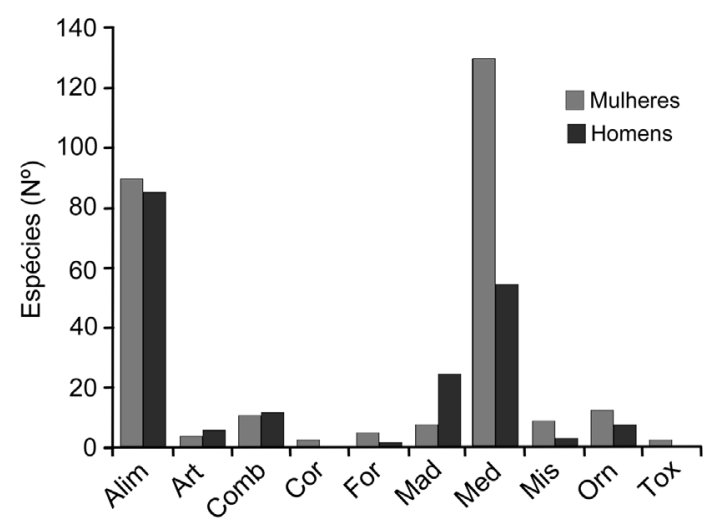

Figura 2 - Número de espécies de plantas, em cada categoria de uso, conhecidas pelos diferentes gêneros de moradores de São Bartolomeu, Ouro Preto, Minas Gerais. (Alim $=$ alimentar, Art $=$ artesanal, $\mathrm{Comb}=$ combustível, Cor $=$ corante, For $=$ forrageira, $\mathrm{Mad}=$ madeireira, Med = medicinal, Mis = mística, Orn = ornamental, Tox $=$ tóxica).

Figure 2 - Number of plant species by each utilitarian category known by the different gender of the community of São Bartolomeu, Ouro Preto, Minas Gerais, Brazil. (Alim = edible, Art $=$ handicraft, Comb $=$ fuel, Cor $=$ dye, For $=$ fodder, Mad $=$ timber, Med $=$ medicinal, Mis = mystical, Orn = ornamental, Tox $=$ toxic). 
representativas em levantamentos etnobotânicos nas regiões de Mata Atlântica (Rossato et al. 1999; Christo et al. 2006; Zuchiwschi et al. 2010) e do Cerrado (Botrel et al. 2006; Moreira \& Guarim-Neto 2009), assim como na transição entre esses domínios (Messias et al. 2015).

Das 232 espécies, 118 são exóticas cultivadas ou ruderais. A preferência pelo uso de plantas exóticas, do ponto de vista da conservação, é considerada negativa, pelo risco de invasão biológica (Poorter \& Ziller 2004). E ainda, para Voeks \& Leoni (2004), caracteriza erosão cultural, onde o uso de plantas exóticas substituiria o das plantas nativas, levando à perda do conhecimento tradicional. Reconhecendo que o conhecimento tradicional é transmitido principalmente pela oralidade através das gerações (Hanazaki 2003; Albuquerque et al. 2010), a premissa de erosão cultural não se aplicaria em comunidades de etnias diversificadas como em São Bartolomeu, com descendentes de europeus, africanos e ameríndios (Queiroz et al. 2013). Desta forma, espera-se que afrodescendentes tenham adquirido conhecimentos de seus ancestrais sobre plantas africanas, assim como descendentes de europeus, sobre as plantas europeias. Além disso, estudos recentes tem discutido o valor das plantas exóticas na manutenção da tradição do uso de plantas pelos povos, sugerindo que a incorporação das mesmas no elenco de plantas úteis seria uma adaptação natural, como produto da evolução do conhecimento sobre os recursos (Hanazaki 2003; Medeiros 2013; Santoro et al. 2015), por muitas vezes suprindo funções inexistentes nas plantas nativas (Albuquerque 2006). Ainda, como ponto positivo, o uso de exóticas com redundância utilitária às espécies nativas poderia reduzir a pressão sobre as mesmas e contribuir com a conservação ambiental (Silva et al. 2014).

Dentre as espécies que apresentam maior valor de uso (Tab. 1, disponibilizada no link $<$ https:// doi.org/10.6084/m9.figshare.7853402.v1>) estão as plantas alimentícias onde se destacam a couve (Brassica oleracea L.), a hortelã (Mentha x villosa Huds.), a goiaba (Psidium guajava L.), a banana, (Musa x paradisiaca L.), o limão [Citrus x limon (L.) Osbeck] e o ora-pro-nobis (Pereskia aculeata Mill.), assim como as medicinais, como a laçavenha (Leonurus japonicus Houtt), transagem (Plantago major L.) e arruda (Ruta graveolens L.). Apenas uma espécie de uso madeireiro, a candeia [Eremanthus erythropappus (DC.) MacLeish], figura entre as 40 plantas com maior valor de uso. Lima et al. (2012), em um estudo etnobotânico no cerrado, encontraram maiores valores de uso para as espécies que apresentavam maior valores de importância fitossociológico. Apesar do presente estudo não contemplar avaliações fitossociológicas, a maioria das espécies com alto valor de uso são cultivadas e ruderais, sugerindo uma relação positiva entre a utilidade e disponibilidade do recurso.

Os habitats mais explorados para obtenção das plantas são as zonas antropizadas, onde são obtidas as espécies ruderais e cultivadas, seguidos pelas florestas. A pouca representatividade de espécies típicas de campos rupestres se justifica pela maior distância dos mesmos, o que torna a obtenção do recurso mais laboriosa. Soldati \& Albuquerque (2012), dentre outros autores, relacionam este aspecto à teoria do forrageamento ótimo, onde o tempo e o esforço são variáveis determinantes no uso dos recursos. Este mesmo princípio explica a maior utilização de espécies cultivadas e ruderais. O grande número de espécies cultivadas revela a tradição agrícola local e reforça a necessidade de maior incentivo para projetos agroecológicos e sustentáveis. Muitos moradores mantêm quintais domésticos, onde empregam métodos de manejo tradicionais. Kumar \& Nair (2004) destacam a longa tradição destas práticas em países tropicais. De acordo com Oakley (2004), os quintais são reservatórios de biodiversidade, reduzem impactos ambientais, garantem a soberania alimentar, fortalecem os vínculos sociais e a preservação da cultura.

Em relação à coleta de plantas na mata, é importante destacar que muitas delas se enquadram dentre os produtos florestais não madeireiros (PFNM). Grande parte dessas espécies são medicinais como a Cecropia hololeuca Miq. (embaúba), Solanum cernuum Vell. (panacéia), Echinodorus grandiflorus (Cham. \& Schltdl.) Micheli (chapéu-de-couro) e Ceiba speciosa (A.St.-Hil.) Ravenna (paineira), das quais se usam as folhas. Outras espécies são comestíveis como Inga edulis Mart. (ingá), Genipa americana L. (jenipapo) e Annona crassiflora Mart. (araticum), das quais se usam os frutos. Brito (2003) relata que a geração de emprego em florestas onde se trabalha com a obtenção de PFNM é de 5 a 15 vezes maior do que com a exploração madeireira. Segundo esta perspectiva, as populações locais seriam mais comprometidas com a conservação se fossem envolvidas e recebessem contrapartidas nesse processo (Berkes 1999; Souza \& Kubo 2006). Todavia, são necessários estudos mais detalhados sobre a disponibilidade dos recursos nos ambientes explorados e a demanda requerida pela comunidade, 
a fim de se estimar níveis sustentáveis de extração de produtos vegetais.

A diversidade de espécies utilizadas por essa comunidade se mostra elevada e comparável a outros levantamentos etnobotânicos no Cerrado e na Mata Atlântica. O valor de H' neste estudo pode ser considerado alto $\left(\mathrm{H}^{\prime}\right.$ base $10=2,16 \mathrm{e} \mathrm{H}$ ' base e $=4,97$ ), quando comparado aos estudos realizados no domínio Atlântico como os de Rossato et al.(1999) $\left(\mathrm{H}_{\text {base } 10}^{\prime}=2,06\right)$; Fonseca-Kruel \& Peixoto (2004) $\left(\mathrm{H}_{\text {base } 10}^{\prime}=1,78 \mathrm{e} \mathrm{H}_{\text {base e }}^{\prime}=4,10\right)$ e Borges \& Peixoto (2009) $\left(\mathrm{H}_{\text {base } 10}^{\prime}=1,81\right)$, e no Cerrado, pelos estudos de Botrel et al. (2006) $\left(\mathrm{H}_{\text {base e }}=\right.$ $4,84)$ e Lima et al. $(2012)\left(\mathrm{H}_{\text {base } 10}=1.13\right.$ a 1.28$)$. Os valores de equabilidade desse estudo $(\mathrm{J}=0,6)$, foram relativamente menores do que nos estudos etnobotânicos citados anteriormente, cujos valores variaram de 0,7 a 0,95 ), indicando que a população analisada tem um conhecimento menos uniforme a respeito do uso de plantas. Muito embora os índices de diversidade em etnobotânica forneçam dados importantes, associando a riqueza e a uniformidade do conhecimento sobre as espécies, é preciso cautela nas comparações entre esses índices nos diversos trabalhos, uma vez que esses valores são sensíveis à amostragem (Magurran 1988).

$\mathrm{O}$ uso medicinal como a categoria de plantas úteis mais citada (Fig. 2) é uma característica comum no domínio dos cerrados (Damasceno \& Barbosa 2008; Moreira \& Guarim-Neto 2009) e da Mata Atlântica (Rossato et al. 1999; Christo et al. 2006; Miranda et al. 2011), sendo as folhas, as partes mais utilizadas. O predomínio do uso de folhas nas preparações medicinais também é comum no cerrado e na mata atlântica (Medeiros 2013), provavelmente pela facilidade de coleta e disponibilidade ao longo do ano. Na perspectiva conservacionista, a utilização de folhas é interessante, já que a sua extração não implicaria, necessariamente, na morte da planta. A tradição do uso de plantas medicinais é uma característica marcante na cultura do município (Messias et al. 2015), o que é reforçado com o presente estudo.

As plantas alimentícias ocupam a segunda categoria com maior riqueza de espécies conhecidas (Fig. 2). Dentre essas, destacam-se pelo valor de uso as espécies produtoras de frutos empregados no feitio dos doces artesanais, principalmente a goiaba, e outros como o figo, banana e pêssego. Há também um grande número de espécies alimentícias ruderais, várias delas reconhecidas como plantas alimentícias não convencionais (PANC's) (Tab. 1, disponibilizada no link < https://doi.org/10.6084/ m9.figshare.7853402.v1>) utilizadas no preparo de pratos típicos, pertencentes à cultura tradicional do município. Dentre essas, destacam o ora-pro-nobis (Pereskia aculeata Mill.), a taioba (Xanthosoma taioba E.G.Gonç.), a samambaia [Pteridium arachnoideum (Kaulf.) Maxon] e a mariaarnica [Erechtites valerianifolius (Wolf) DC.]. O conhecimento e uso das PANC's contribui para aumentar a agrobiodiversidade e permite a diversificação e melhoria da dieta (Kinupp \& Barros 2008). Essas espécies possuem ainda grande potencial econômico, visto que o turismo é uma das principais atividades econômicas do município, que também usa a gastronomia como um de seus atrativos.

Com relação às plantas alimentares olerícolas que eram tradicionalmente cultivadas no distrito, como o alho e a baroa, há a necessidade de programas de assistência técnica aos agricultores visando o manejo agroecológico ou controle biológico da doença causada por Sclerotium spp. O uso de tecnologias agroecológicas para o controle dessa doença, que é de difícil controle por métodos químicos tradicionais, mostra-se eficiente (Michereff et al. 2005; Lohmann et al. 2007), devendo ser estimulado nesse local. A restauração desses sistemas produtivos contribuiria para a diversificação da agricultura local e da soberania alimentar da comunidade. De acordo com os entrevistados, nenhuma das plantas citadas com fins madeireiros é extraída no presente. Tal fato está relacionado com a legislação imposta pela gestão da UC. Os entrevistados mais idosos ainda conhecem espécies madeireiras úteis na construção de casas, mobiliário e cercas, mas pela falta de uso dessas plantas, esse conhecimento tenderia a se perder ao longo do tempo. Algumas espécies madeireiras nativas utilizadas em cabos de ferramentas, bengalas e instrumentos, cujos usos são pouco conhecidos, foram também mencionadas (Tab. 1, disponibilizada no link <https://doi.org/10.6084/ m9.figshare.7853402.v1>).

Dentre as plantas úteis, apenas as espécies utilizadas pelos moradores locais como combustíveis podem representar algum impacto, visto a larga utilização da lenha em fornalhas, para a confecção dos doces tradicionais artesanais. No entanto, os moradores entrevistados relataram que sempre conviveram com a extração da lenha na mata que circunda o povoado de São Bartolomeu, sem provocar impactos com essa atividade. Nas entrevistas foram descritos que durante a extração, prioriza-se a utilização de galhos secos, árvores mortas ou de 
espécies muito abundantes que fornecem boa lenha, como a candeia (Eremanthus erythropappus (DC.) MacLeish). Diversos moradores relataram que tentativas de doação de lenha por órgãos ambientais e gestores da UC, como estratégia para combater o extrativismo, geraram conflitos na comunidade por discórdias quanto à sua distribuição. Desde a criação da APA esse tem sido o principal conflito de uso, carecendo ainda de soluções sustentáveis.

Menores proporções de plantas úteis em categorias que não sejam medicinais, alimentares e madeireiras, também são mostradas em outros estudos etnobotânicos (Christo et al. 2006; Damasceno \& Barbosa 2008; Moreira \& GuarimNeto 2009). A pouca utilização de plantas místicas no distrito (Fig. 2) provavelmente se relaciona aos aspectos religiosos dos moradores, que são, na maioria, católicos. Mesmo assim, plantas nativas, africanas e europeias são usadas para atrair sorte e dinheiro, afastar mal olhado e para rituais de benzeção, pelas benzedeiras da comunidade. A riqueza de plantas ornamentais é pequena (Fig. 2), sendo grande parte exótica e utilizadas em jardins e quintais. E ainda, poucos entrevistados têm criação de animais, o que explica o baixo número de espécies citadas para forragem. Dentre as poucas plantas tóxicas citadas, a maioria é utilizada como inseticida ou repelente contra insetos. O uso de plantas para artesanato, assim como de corantes (todas são utilizadas para corar fibras artesanais) também apresentou baixa expressividade (Fig. 2). As espécies utilizadas para artesanato são usadas para fazer cestos, esteiras e ornamentos. O artesanato não é uma atividade típica do distrito, explicando o pouco conhecimento de plantas nessas categorias. Todavia, com o aumento do turismo na região, o estímulo à produção de peças artesanais empregando partes de plantas e corantes naturais poderia ser uma atividade econômica que melhoraria a geração de renda local.

Nove espécies citadas são referidas como ameaçadas de extinção (Tab. 1, disponibilizada no link $<$ https://doi.org/10.6084/m9.figshare.7853402. v1 $>$ ). No entanto, apenas a espécie medicinal conhecida como arnica (Lychnophora pinaster Mart.) é mencionada com uso atual. Porém, o seu uso é relatado como raro, dado a distância do distrito às áreas de ocorrência dessa espécie.

\section{Relações entre o uso das plantas}

e características socioeconômicas

$\mathrm{O}$ conhecimento a respeito das plantas úteis varia de acordo com o gênero $(\mathrm{G}=35.699$, g.l. $=9, \mathrm{p}<$ 0,0001). Em média, as mulheres citaram 14 espécies medicinais, enquanto que os homens, apenas seis. Por outro lado, os homens conhecem mais plantas madeireiras (em média 6) que mulheres (média = 0,3) (Fig. 2). Outros estudos tem apontado resultados semelhantes, indicando uma divisão de tarefas e de conhecimentos tradicionais sobre as plantas úteis (Borges \& Peixoto 2009; Giraldi \& Hanazaki 2010; Viu et al. 2010). Kainer \& Duryea (1992) e Voeks (2007) relatam que o contexto histórico do papel feminino possibilita maior conhecimento sobre plantas medicinais, alimentares, herbáceas e exóticas. As demais características socioeconômicas analisadas (idade, escolaridade, ocupação, tempo de moradia e renda) não apresentaram diferenças significativas em relação à riqueza de espécies vegetais conhecidas $(\mathrm{p}>0,05)$.

Alguns estudos etnobotânicos têm observado que as pessoas mais idosas da comunidade possuem maior conhecimento sobre plantas (Hanazaki et al. 2000; Fonseca-Kruel \& Peixoto 2004). A idade dos 43 entrevistados nesse estudo variou entre 18 e 89 anos, onde foram entrevistados 5 jovens ( $<$ 25 anos), 5 pessoas entre 25 e 44 anos, 18 entre 45 e 64 anos e 15 pessoas acima de 65 anos. Embora não haja diferenças significativas no número de espécies conhecidas pelos moradores de diferentes faixas etárias $(\mathrm{G}=5,57, \mathrm{~g} .1 .=3, \mathrm{p}=0,13)$, verificouse que os entrevistados mais jovens $(<25$ anos) conhecem, em média, 16 espécies, enquanto que os mais idosos (45 a 65 anos e $>65$ anos), conhecem em média, 35 e 28 espécies, respectivamente. Este fato sugere a implementação de práticas educativas favorecendo a disseminação dos conhecimentos etnobotânicos para a população mais jovem. O menor conhecimento dos mais jovens sugerem, de acordo com Palmer (2004), certa erosão do conhecimento ou aculturação. No entanto, esta interpretação deve ser vista com parcimônia uma vez que o acúmulo de conhecimentos tradicionais se dá ao longo da vida pelas experiências e vivências (Sousa et al. 2012). Outro fato que pode ter determinado o menor conhecimento de espécies pelos moradores mais jovens é a limitação ao uso das espécies nativas imposta pela gestão da UC, uma vez que o conhecimento ecológico tradicional é construído pela relação do homem com o meio ambiente (Zuchiwschi et al. 2010). A população jovem (até 25 anos) é pouco representativa (11,9\%) quando comparada à média do Brasil (20\%) (IBGE 2010). Pelos relatos apresentados isto se deve às reduzidas opções de emprego e renda, o que leva os indivíduos mais jovens emigrarem em busca de oportunidades de trabalho (Prado 2014). Programas 
de estímulo à utilização sustentável de recursos poderiam permitir, além da melhoria da qualidade de vida, oportunidade de trabalho para a comunidade e manutenção da cultura.

Dentre as variáveis socioeconômicas avaliadas, a renda, escolaridade, tempo de moradia no local e ocupação apresentaram pouca variação entre os entrevistados, justificando a não influência das mesmas na quantificação do conhecimento tradicional. A renda per capita do distrito é baixa, $50 \%$ dos entrevistados ganham até 1 salário mínimo e $84 \%$ deles recebem até 2 salários mínimos. A escolaridade também apresentou pouca variação, onde cerca de $72 \%$ dos entrevistados cursaram até o ensino fundamental. A maioria dos entrevistados é oriunda de famílias que ocupam o território por várias gerações, com ocupação primária ou secundária relacionada, direta ou indiretamente ao uso dos recursos naturais.

\section{Uso sustentável dos recursos}

A localidade vem passando, nos últimos anos, por várias alterações que podem levar à descaracterização e perda do conhecimento tradicional sobre a biodiversidade. Este conhecimento pode e deve ser usado para conservar e aumentar a diversidade biológica (Giraldi \& Hanazaki 2010). $\mathrm{O}$ incremento na procura turística, a chegada de moradores com características diferentes na comunidade e a existência de restrições em relação ao uso da terra são fatores que influenciam a cultura local, gerando a inclusão de novas características, bem como a perda de outras.

Projetos de extrativismo de PFNM são passíveis de ser implantados na Zona de Manejo Florestal e Faunístico na FLOE do Uaimií. O Plano de Manejo da FLOE prevê o desenvolvimento de ações para possibilitar o manejo comunitário, estando a área incluída na Rede Ibero-americana de Bosques Modelo (IEF 2011). As florestas ou bosques modelo têm a finalidade de promover a conservação e a utilização sustentável dos recursos florestais pelas comunidades locais.

Pela tradição agrícola e de fabricação de doces artesanais, há a necessidade de incentivar e garantir a produção autossuficiente de frutas. Adicionalmente, estudos posteriores com relação ao extrativismo de lenha são necessários para averiguar a disponibilidade do recurso em face das demandas locais e impactos potenciais frente a um possivel aumento da demanda de lenha. De acordo com os relatos apresentados, este é o principal ponto de conflito entre a comunidade e as diretrizes da UC. Faz-se necessário fortalecer os canais de diálogo e mediação a fim de promover iniciativas de redução dos conflitos, garantir o desenvolvimento social e econômico e preservação dos recursos naturais e culturais de São Bartolomeu.

As diretrizes estabelecidas para as UCs de uso sustentável têm como objetivo compatibilizar o uso dos recursos com a conservação da natureza (Diegues 2004). Dessa forma, políticas públicas precisam ser formuladas para a garantia do papel dessa UC, como, por exemplo, estratégias para a geração de emprego e renda, uma vez que o êxodo tem sido marcado, principalmente pela população jovem (Scalco \& Gontijo 2009). Acredita-se que a valorização do conhecimento sobre as plantas, o investimento em práticas agroecológicas e o uso sustentável da flora serão úteis para o desenvolvimento socioeconômico e ambiental da região, fortalecendo a identidade e a autonomia dos moradores.

\section{Agradecimentos}

Os autores agradecem à comunidade de São Bartolomeu, a receptividade e compartilhamento de saberes; à CAPES, a bolsa de Mestrado concedida à primeira autora; e aos revisores, as valiosas contribuições ao manuscrito.

\section{Referências}

Albuquerque UP (2006) Re-examining hypotheses concerning the use and knowledge of medicinal plants: a study in the Caatinga vegetation of $\mathrm{NE}$ Brazil. Journal of Ethnobiology and Ethnomedicine 2: 30 .

Albuquerque UP, Lucena RFP \& Cunha LVFC (2010) Métodos e técnicas na pesquisa etnobiológica e etnoecológica. $3^{\text {a }}$ ed. NUPEEA, Recife. 559p.

Álvares CA, Stape JL, Sentelhas PC, Gonçalves JLM \& Sparovek G (2013) Koppen's climate classification map for Brazil. Meteorologische Zeitschrift 22: 711-728.

Arias-Toledo B, Colantonio S \& Galetto L (2007) Knowledge and use of edible and medicinal plants in two populations from the Chaco forest, Córdoba Province, Argentina. Journal of Ethnobiology 27: 218-232.

Berkes F (1999) Sacred ecology: traditional ecological knowledge and management systems. Taylor \& Francis, Philadelphia, London. 209p.

Borges R \& Peixoto AL (2009) Conhecimento e uso de plantas em uma comunidade caiçara do litoral sul do estado do Rio de Janeiro, Brasil. Acta Botanica Brasilica 23: 769-779.

Botrel RT, Rodrigues LA, Gomes LJ, Carvalho DA \& Fontes MAL (2006) Uso da vegetação nativa pela população local no município de Ingaí, MG, Brasil. Acta Botanica Brasilica 20: 143-156. 
Brito JO (2003) Produtos florestais não-madeireiros: um importante potencial nas florestas. Informativo ARESB. Disponível em <http://www.ipef.br/ tecprodutos/aresb001.asp $>$. Acesso em 25 março 2015.

Campos LZ, Albuquerque UP, Peroni N \& Araújo EL (2015) Do socioeconomic characteristics explain the knowledge and use of native food plants in semiarid environments in Northeastern Brazil? Journal of Arid Environments 115: 53-61.

Chase MW \& Reveal JL (2009) A phylogenetic classification of the land plants to accompany APG III. Botanical Journal of the Linnean Society 161: 122-127.

Christo G, Guedes-Bruni RR \& Fonseca-Kruel VS (2006) Uso de recursos vegetais em comunidades rurais limítrofes à Reserva Biológica do Poço das Antas, Silva Jardim, Rio de Janeiro: estudo de caso na gleba Aldeia Velha. Rodriguésia 57: 519-542.

COPAM (1997) Deliberação COPAM no 85, 30 de outubro de 1997. 1 cad. Conselho Estadual de Política Ambiental, Belo Horizonte. Pp. 10-12.

Damasceno SS \& Barbosa AAA (2008) Levantamento etnobotânico de plantas do bioma cerrado na comunidade de Martinésia, em Uberlândia, Minas Gerais. Horizonte Científico 8: 1-30.

Diegues ACS (2004) O mito moderno da natureza intocada. $4^{\mathrm{a}}$ ed. HUCITEC/NUPAUB, São Paulo. 176p.

Diegues ACS \& Viana VM (2004) Comunidades tradicionais e manejo dos recursos naturais da Mata Atlântica. $2^{a}$ ed. HUCITEC/NUPAUB, São Paulo. $273 p$.

Fonseca-Kruel VS \& Peixoto AL (2004) Etnobotânica na Reserva Extrativista Marinha de Arraial do Cabo, RJ, Brasil. Acta Botanica Brasilica 18: 177-190.

Giraldi M \& Hanazaki N (2010) Uso e conhecimento tradicional de plantas medicinais no Sertão do Ribeirão, Florianópolis, SC, Brasil. Acta Botanica Brasilica 24: 395-406.

González JA, García-Barriuso M \& Amich F (2011) The consumption of wild and semi-domesticated edible plants in the Arribes del Duero (Salamanca-Zamora, Spain): an analysis of traditional knowledge. Genetic Resources and Crop Evolution 58: 991-1006.

Hanazaki N, Tamashiro JY, Leitão-Filho HF \& Begossi A (2000) Diversity of plant use in two caiçara communities from the Atlantic Forest coast, Brazil. Biodiversity \& Conservation 9: 597-615.

Hanazaki N (2003) Comunidades, conservação e manejo: o papel do conhecimento ecológico local. Biotemas 16: $23-47$.

Hegde R \& Enters T (2000) Forest products and household economy: a case study from Mudumalai Wildlife Sanctuary, Southern India. Environmental Conservation 27: 250-259.

IBGE (1983) Metodologia do censo demográfico de 1980. Vol. 4. Fundação Instituto Brasileiro de Geografia e Estatística - IBGE, Rio de Janeiro. 478p.
IBGE (2010) Censo demográfico 2010. Disponível em <http://www.censo2010.ibge.gov.br/dados divulgados/index.php? $u f=31>$. Acesso em 24 junho 2012.

IEF - Instituto Estadual de Florestas (2006) Relatório de zoneamento ecológico econômico da área de proteção ambiental Cachoeira da Andorinhas. Universidade Federal de Viçosa, Viçosa. 107p.

IEF - Instituto Estadual de Florestas (2011) Plano de manejo da Floresta Estadual do Uaimií. Encarte 1: diagnóstico da floresta estadual do Uaimií. Universidade Federal de Viçosa, Viçosa. 184p.

IUCN (2015) The IUCN Red List of Threatened Species. Version 2015-4. Disponível em <http://www. iucnredlist.org>. Acesso em 12 novembro 2015.

Kainer KA \& Duryea ML (1992) Tapping women's knowledge: plant resource use in Extractive Reserves, Acre, Brazil. Economic Botany 46: 408-425.

Kinupp VF \& Barros IBI (2008) Teores de proteína e minerais de espécies nativas, potenciais hortaliças e frutas. Ciência e Tecnologia de Alimentos 28: 846-857.

Kumar BM \& Nair PR (2004) The enigma of tropical home gardens. Agroforestry Systems 61: 135-152.

Lima ILP, Scariot AMMB \& Sevilha AC (2012) Diversidade e uso de plantas do Cerrado em comunidade de Geraizeiros no norte do estado de Minas Gerais, Brasil. Acta Botanica Brasilica 26: 675-684.

Lohmann TR, Pazuch D, Stangarlin JR, Selzlein C \& Nacke H (2007) Seleção de isolados de Trichoderma spp. para controle de Sclerotium rolfsii em soja. Revista Brasileira de Agroecologia 2: 1665-1668.

Magurran A (1988) Ecological diversity and its measurement. Croom-Helm, London. 192p.

Maia-Neto GA (2009) Unidades de conservação de uso sustentável. Revista Jus Navigandi 14(2342). Disponível em <https://jus.com.br/artigos/13933> Acesso em 15 março 2017.

Medeiros PM (2013) Why is change feared? Exotic species in traditional pharmacopoeias. Ethnobiology and Conservation 2: 1-5.

Messias MCTB, Menegatto MFM, Prado ACC, Santos BR \& Guimarães MFM (2015) Uso popular de plantas medicinais e perfil socioeconômico dos usuários: um estudo em área urbana em Ouro Preto, MG, Brasil. Revista Brasileira de Plantas Medicinais 17: 76-104.

Michereff SJ, Andrade DEGT \& Menezes M (2005) Ecologia e manejo de patógenos em solos tropicais. Imprensa Universitária UFRPE, Recife. 398p.

Miranda TM, Hanazaki N, Govone JS \& Alves DMM (2011) Existe utilização efetiva dos recursos vegetais conhecidos em comunidades caiçaras da Ilha do Cardoso, estado de São Paulo, Brasil? Rodriguésia 62: 153-169.

MMA - Ministério do Meio Ambiente (2004) Sistema nacional de unidades de conservação da natureza: Lei $n^{\circ} 9.985$, de 18 de julho de 2000; Decreto $n^{\circ}$ 
4.340, de 22 de agosto de 2002. 5a ed. MMA/SBF, Brasília. 56p.

MMA - Ministério do Meio Ambiente (2014) Lista nacional oficial de espécies da flora ameaçadas de extinção. Portaria $n^{\circ} 443$, de 17 de dezembro de 2014. Disponível em <http://pesquisa.in.gov.br/ imprensa/jsp/visualiza/index.jsp?data $=18 / 12 / 201$ $4 \&$ jornal $=1 \&$ pagina $=110 \&$ totalArquivos $=144>$. Acesso em 15 fevereiro 2015.

Moreira DL \& Guarim-Neto G (2009) Usos múltiplos de plantas do cerrado: um estudo etnobotânico na comunidade Sítio Pindura, Rosário Oeste, Mato Grosso, Brasil. Polibotánica 27: 159-190.

Oakley E (2004) Quintais Domésticos: uma responsabilidade cultural. Agriculturas 1: 37-39.

Ostrom E (1990) Governing de commons: the evolutions of institutions for collective actions. Cambridge University Press, Cambridge. 280p.

Palmer C (2004) The inclusion of recently introduced plants in the Hawaiian ethnopharmacopoeia. Economic Botany 58: S280-S293.

Poorter M \& Ziller SR (2004) Biological contamination in protected areas: the need to act and turn the tide of invasive alien species. In: Milano MS, Takahashi LY \& Nunes ML (eds.) Unidades de conservação: atualidades e tendências. Fundação O Boticário de Proteção à Natureza, Curitiba. Pp. 118-131.

Prado ACC (2014) Estudo etnobotânico com vistas à sustentabilidade local do distrito de São Bartolomeu, Ouro Preto, MG. Dissertação de Mestrado em Ecologia de Biomas Tropicais. Universidade Federal de Ouro Preto, Ouro Preto. $114 \mathrm{p}$.

Queiroz EM, Santos AM, Castro IM, Machado-Coelho GL, Cândido AP, Leite TM, Pereira RW \& Freitas RN (2013) Genetic composition of a Brazilian population: the footprint of the Gold Cycle. Genetics and Molecular Research 12: 5124-5133.

Rossato SC, Leitão-Filho HF \& Begossi A (1999) Ethnobotany of Caiçaras of the Atlantic Forest Coast (Brazil). Economic Botany 53: 387-395.

Santoro FR, Ferreira Júnior WS, Araújo TAS, Ladio AH \& Albuquerque UP (2015) Does plant species richness guarantee the resilience of local medical systems? A Perspective from utilitarian redundancy. Plos One 10: e0119826.

Scalco RF (2009) Desafios, paradoxos e complexidade na gestão do mosaico de unidades de conservação da área de proteção ambiental Cachoeira das Andorinhas, Ouro Preto, MG. Dissertação de
Mestrado. Universidade Federal de Minas Gerais, Belo Horizonte. 228p.

Scalco RF \& Gontijo BM (2009) Mosaico de unidades de conservação: da teoria à prática. $\mathrm{O}$ caso do mosaico de unidades de conservação da APA Cachoeira das Andorinhas, Ouro Preto/MG. Geografias 5: 75-92.

Silva FS, Albuquerque UP, Costa Júnior LM, Lima AS, Nascimento ALB \& Monteiro JM (2014) An ethnopharmacological assessment of the use of plants against parasitic diseases in humans and animals. Journal of Ethnopharmacology 155: 1332-1341.

Soldati GT \& Albuquerque UP (2012) A new application for the optimal foraging theory: the extraction of medicinal plants. Evidence-Based Complementary and Alternative Medicine 2012: 1-10.

Sousa R, Hanazaki N, Lopes JB \& Barros RM (2012) Are gender and age important in understanding the distribution of local botanical knowledge in fishing communities of the Parnaiba Delta Environmental Protection Area? Ethnobotany Research and Applications 10: 551-559.

Souza GC \& Kubo R (2006) A perspectiva da etnobotânica sobre o extrativismo de produtos florestais não madeiráveis e a conservação. In: Kubo R, Bassi JB, Souza GPC, Alencar NL, Medeiros PM \& Albuquerque UP (eds.) Atualidades em etnobiologia e etnoecologia. Vol. 3. Nupeea/ SBEE, Recife. Pp. 85-98.

Tardio J \& Santayana MP (2008) Cultural importance indices: a comparative analysis based on the useful wild plants of Southern Cantabria (Northern Spain). Economic Botany 62: 24-30.

Viu AFM, Viu MAO \& Campos LZO (2010) Etnobotânica: uma questão de gênero? Revista Brasileira de Agroecologia 5: 138-147.

Voeks RA (2007) Are women reservoirs of traditional plant knowledge? Gender, ethnobotany and globalization in northeast Brazil. Journal of Tropical Geography 28: 7-20.

Voeks RA \& Leony A (2004) Forgetting the forest: assessing medicinal plant erosion in eastern Brazil. Economic Botany 58: S294-S306.

Zar JH (1996) Biostatistical Analysis. $3^{\text {rd }}$ ed. Prentice Hall, New Jersey. 944p.

Zuchiwschi E, Fantini AC, Alves AC \& Peroni N (2010) Limitações ao uso de espécies florestais nativas pode contribuir com a erosão do conhecimento ecológico tradicional e local de agricultores familiares. Acta Botanica Brasilica 24: 270-282. 